\title{
Simulation Study on Geometric Anisotropic Estimators for Spatial Point Process
}

\author{
Williams Kumi \\ Department of Mathematics \& Statistics, School of Science, University of Energy \& Natural Resources, Sunyani, Ghana \\ Email: Williams.kumi@uenr.edu.gh
}

How to cite this paper: Kumi, W. (2021) Simulation Study on Geometric Anisotropic Estimators for Spatial Point Process. Open Access Library Journal, 8: e8095. https://doi.org/10.4236/oalib.1108095

Received: October 19, 2021

Accepted: November 21, 2021

Published: November 24, 2021

Copyright $\odot 2021$ by author(s) and Open Access Library Inc.

This work is licensed under the Creative Commons Attribution International License (CC BY 4.0).

http://creativecommons.org/licenses/by/4.0/

\section{(c) (i) Open Access}

\begin{abstract}
The method by Fry for detecting geometric anisotropy in stationary spatial point pattern is investigated. We quantify anisotropy by stretching and compressing the point process about the axis. Using a simulated Strauss point pattern, we first fit ellipsoids to the compressed pattern of pairwise difference vectors to estimate the direction of anisotropy. The strength of compression and the regularity of the point process are varied at different times and the corresponding effect on the estimated ellipsoids is investigated as the level of contour ellipsoid increases. Parameters and contours good for estimation were discussed.
\end{abstract}

\section{Subject Areas}

Mathematical Statistics

\section{Keywords}

Contour Ellipsoids, Geometric Anisotropy, Pairwise Difference Vectors, Strauss Point Pattern

\section{Introduction}

Stationarity and isotropy (translation and rotational invariance of the distribution) have been the assumptions of spatial point pattern that catch the attention of many readers. However, anisotropy has not gotten much attention. The Fry plot gives more idea of anisotropy by creating an empty space of ellipse at the center if the point process is regular, for clustered process, the empty space turns out to be black depending on the shape of the clusters [1] [2].

The main task of this paper is to evaluate geometric anisotropy estimators for spatial point patterns. The method used is the Fry plot method, thus the plot of pairwise difference vectors of all points of the process. We will create a series of 
sectors (from point process) beaming out of the origin in a specified direction and count the number of points falling into each of these sectors [1]. We will then compress and stretch the process about these axes and also rotate the process about the origin. Finally, we will fit ellipses to the compressed point process. The first ellipse we estimate represents the first level of contour. In our simulation study, we will perform a statistical test ( $\mathrm{P}$-value test of equality of the semi axes) and explain how variation in the parameters affects the semi axes of the estimated ellipse as the level of contours increase. Again, we will also discuss a measure called rotangle which checks the direction of compression.

\section{Notations}

$X$ is assumed to be a stationary and isotropic point process; for a Borel set; $B \subseteq R^{d}$, let $\mu$ be the Lebesgue measure on the Borel set $B$. See ([1] [3]). Let;

$$
N_{X}(B):=\sum_{x \in X} 1_{B}(x)
$$

be the counting measure defined by $X$ and $v(B)=\mu(B)$ be the volume of $B$.

Additionally, we assume that the point pattern observed is of the form;

$$
y:=\left\{y_{i} ; y_{i} \in W, i=1, \cdots, n\right\}
$$

$W \subset R^{d}$ (where usually $d=2$ or $d=3$ in applications) is the observation window and comes from the transformed process;

$$
Y:=T X=\{T x ; x \in X\}
$$

where $T: R^{d} \rightarrow R^{d}$ is an invertible linear mapping. Due to isotropy of $X$, we can split the mapping into two matrices (see [1]).

$$
T=R C
$$

where $R$ is the rotation matrix that rotates the point process about the origin and $C$ is the diagonal scaling matrix for compression and stretching of dimensions.

\subsection{Estimation of the Rotation; $R$}

Let;

$$
F(Y):=\left\{f_{i j}=y_{i}-y_{j} ; y_{i}, y_{j} \in Y\right\}
$$

be the Fry points in $R^{d}$ (see [1] and [4]). Under this method of estimating $R$, the Fry points are also transformed under $T$. Let $\rho^{(2)}$ be the second order product density of $X$ and assume that it is continuous and monotone. No directional dependence exists under isotropy so the contours of the form;

$$
l_{l}:=\left\{(r, u) \in R_{+} \times S^{d-1} ; r=\arg \min _{t}\left\{\rho^{(2)}(t u)=l\right\}\right\}
$$

are spheres where $l^{\prime} s$ are the range of $\rho^{(2)}$ and $u$ is the direction vector [1]. Under the linear transformations, these sets become ellipsoids. Now they defined the counting measure 


$$
N_{Y}(u, r)=\sum_{f_{i j} \in F(Y)} 1\left(f_{i j} \in S_{\varepsilon}(u, r)\right)
$$

which counts the Fry points within this r-sector. Let the distance

$$
r_{l}(u):=\arg \min _{t}\left\{\left(N_{Y}(u, t)\right)=l\right\}
$$

for $l=1,2, \cdots$ defines the minimum distance in the sector $S_{\varepsilon}(u)$ having $l$ points.

\subsection{Estimating Scaling}

Thomas A. et al. (2016) [1] assumed that the data is back rotated $\tilde{y}=\hat{R}^{-1} y \approx C x$ which conforms approximately to the original isotropic point pattern with some invertible diagonal scaling matrix $C$ [3] with the assumption that the volume of the spheroidal transformation is preserved, $C=\operatorname{diag}\left(c^{-\frac{1}{2}}, c^{-\frac{1}{2}}, c\right)$. i.e. only one free parameter; $0<c<1$ which compresses the pattern top to down.

\section{Simulation Study}

In our simulation study, we considered a stationary Strauss process with fixed number of points at $n=300$ and the interaction radius were fixed at $r=0.04$. We then varied the regularity parameter between $\gamma=0.0$ (most regular case), $\gamma=0.1$ and $\gamma=0.3$ (least regular case). Concerning the parameters for the Fry plot for estimating anisotropy, we varied the strength of compression from $c=$ $0.5,0.6,0.7,0.8,0.9,1.0$ with the compression model $\left(\frac{1}{c}, c\right)$ for compression in the $\mathrm{y}$-direction and dilation in the $\mathrm{x}$-direction. The number of sectors were fixed at angles $=50$ and for the observation window, we considered a unit cube $[0,1] \times[0,1]$. For the level of contours, we considered from 1 up to 20 . However, we extented the contours up to 30 in our further simulations to confirm our observation for higher level of contours. We simulated 100 times.

\subsection{Rotangle}

The rotangle is the angle which checks the direction of compression. Since the compression was done vertically, then we expect to get rotation angle around $\frac{\pi}{2}$.

If the compression is diagonal (anticlockwise), then you should get rotangle $=\frac{\pi}{4}$.

\subsubsection{Rotangle, Fixing Gamma and Varying Compression for One} Simulation

We started by fixing the regularity parameter, $\gamma$ and the strength of compression was being varied for only one simulation. We plotted the rotangle against the contours. The marked rotangle was indicated with black horizontal line at $\frac{\pi}{2}$ and different colours were chosen for different strength of compressions. Figure 1 below summarises our results. 

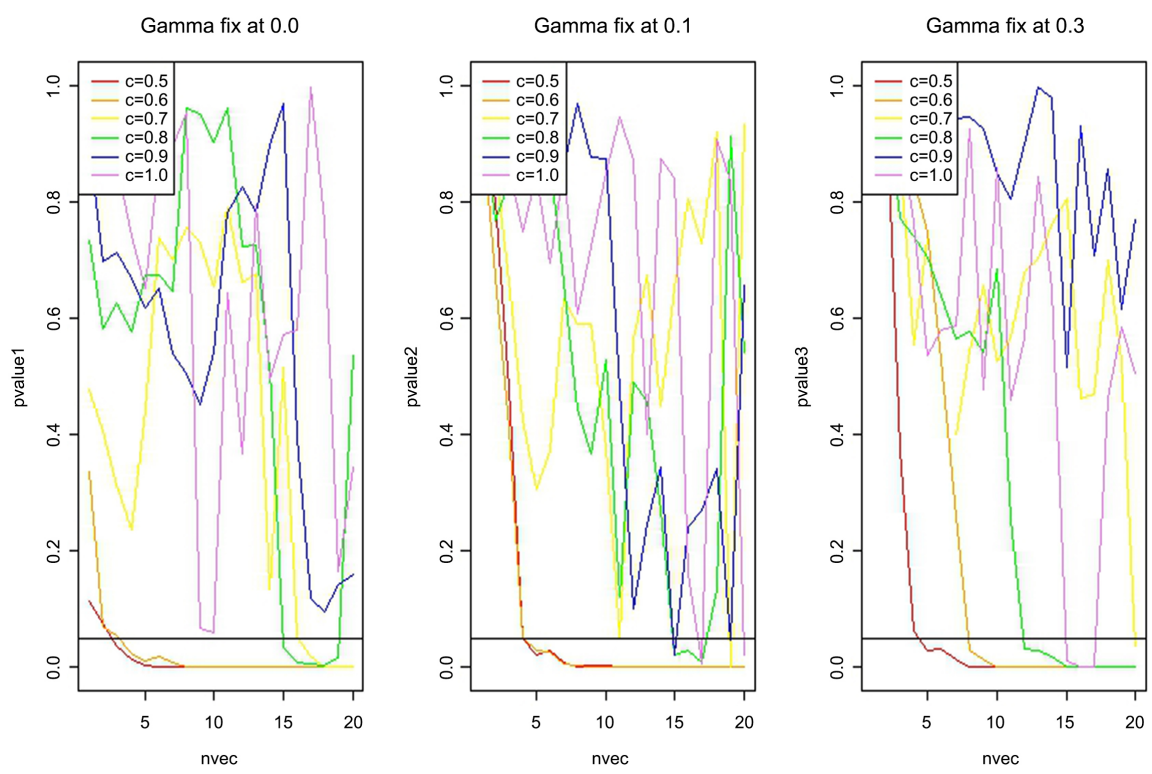

Figure 1. Rotangle against contours for fixed gamma and varying compression for one simulation.

\subsubsection{Interpretation for Figure 1}

From our plot, when gamma was fixed at 0.0 , only $c=0.5$ and $c=0.6$ were the parameters for which the rotangle were close to the marked rotangle at $\frac{\pi}{2}$.

From $c=0.7$ up to 1.0 the rotangle deviates from the marked rotangle and hence the contours are not informative. The higher levels of contour are more informative than the lower contours.

\subsubsection{Rotangle, Fixing Compression and Varying Gamma for One Simulation}

Now we fixed the strength of compression and the regularity parameter was being varied for only one simulation. Figure 2 below illustrates our results.

\subsubsection{Interpretation for Figure 2}

We observed some breakage when compression was fixed at $c=0.5(\gamma=0.0)$ because some values were missing. From the plot, we observed that the contours were informative at the instances where the compression was higher, thus when compression was fixed at $c=0.5,0.6,0.7$. At low strength of compression, the contours look more spherical like expected. The worst case was when there was no compression at $c=1.0$. As the strength of compression increases, then the contours become more elliptical.

\subsubsection{Rotangle, Fixing Gamma and Varying Compression for 100 Simulations}

Now we fixed gamma and compression was being varied. We chose the contour levels $I=5,10,15,20,25,30$ due to higher number of contours and the simulation was done 100 times. The box plot in Figure 3 below summarises our results. 
comp fix at 0.5

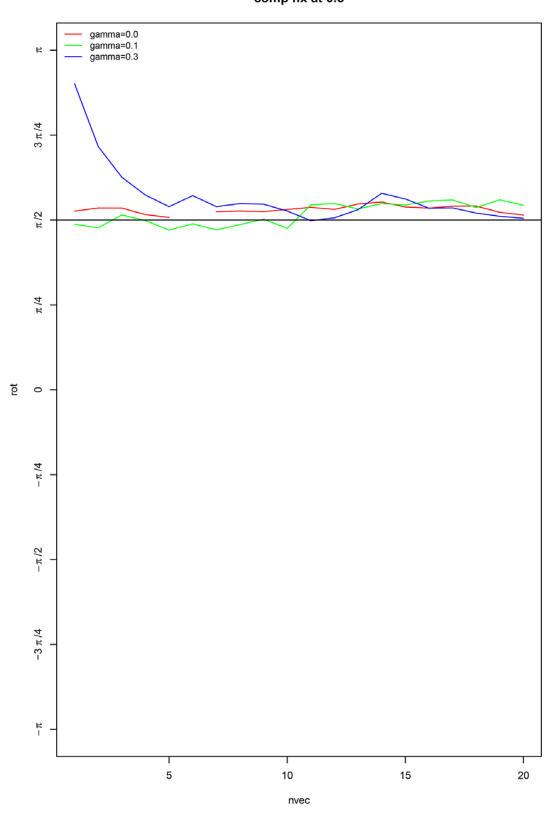

comp fix at 0.8

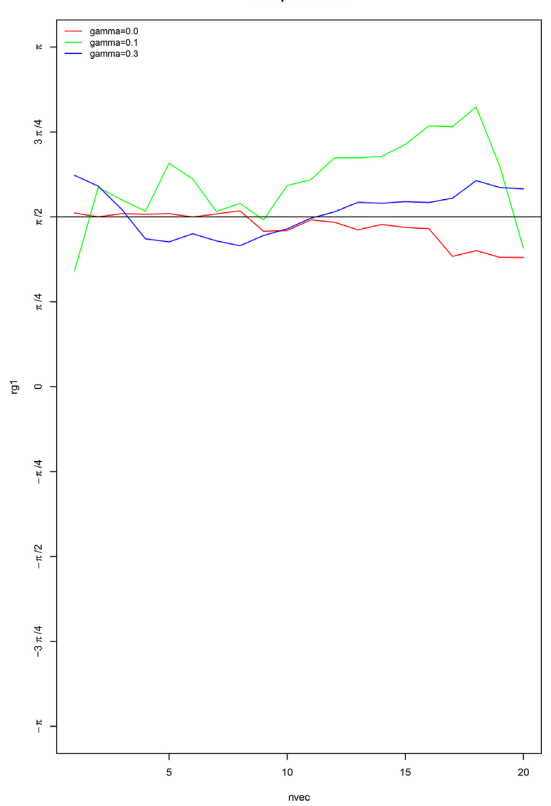

comp fix at 0.6

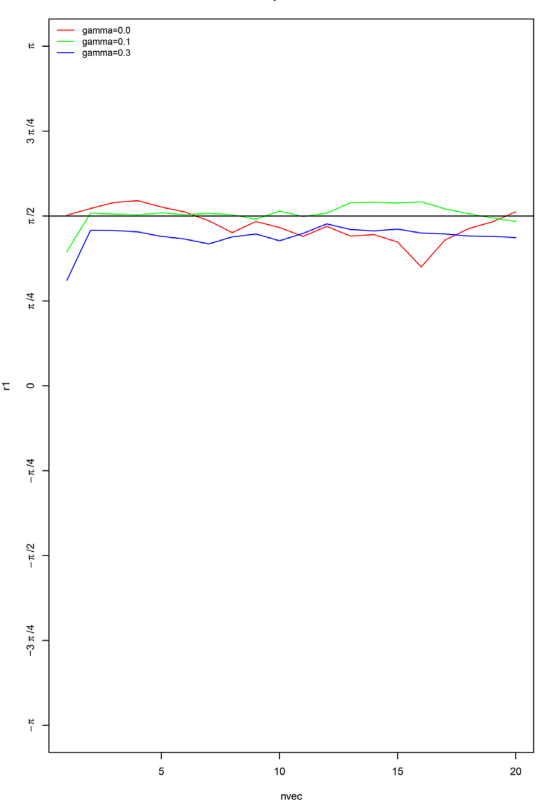

comp fix at 0.9

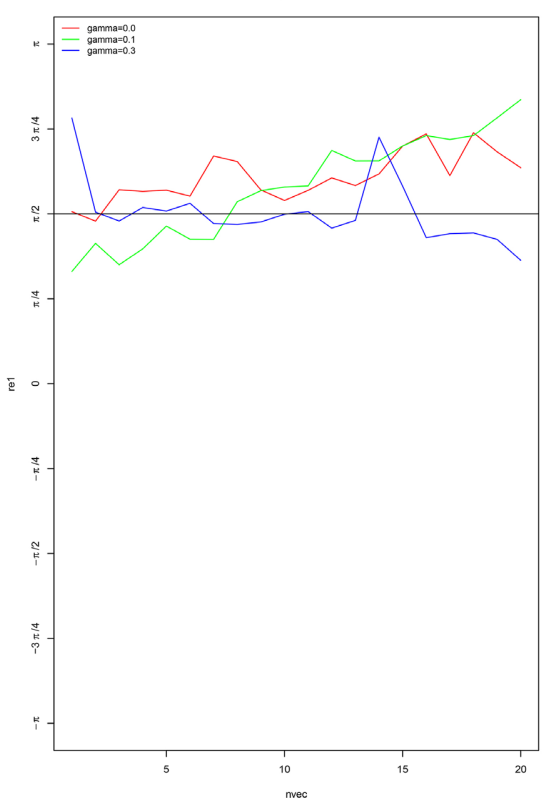

comp fix at 0.7

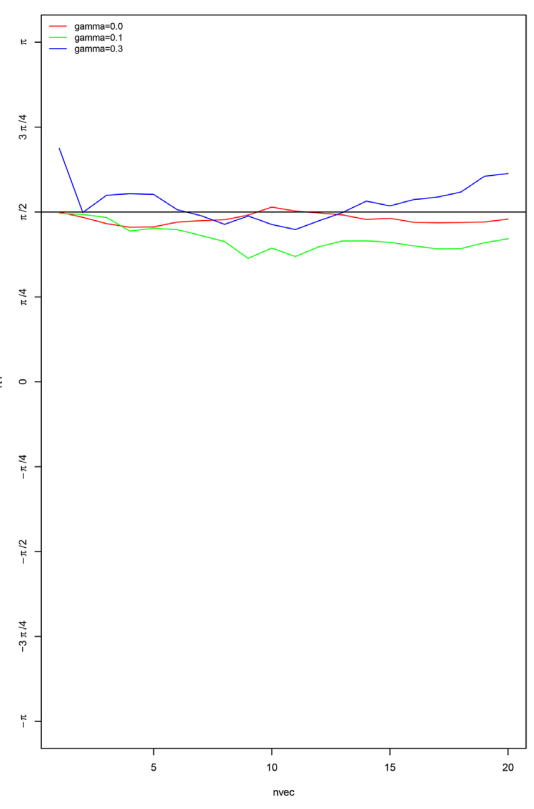

comp fix at 1.0

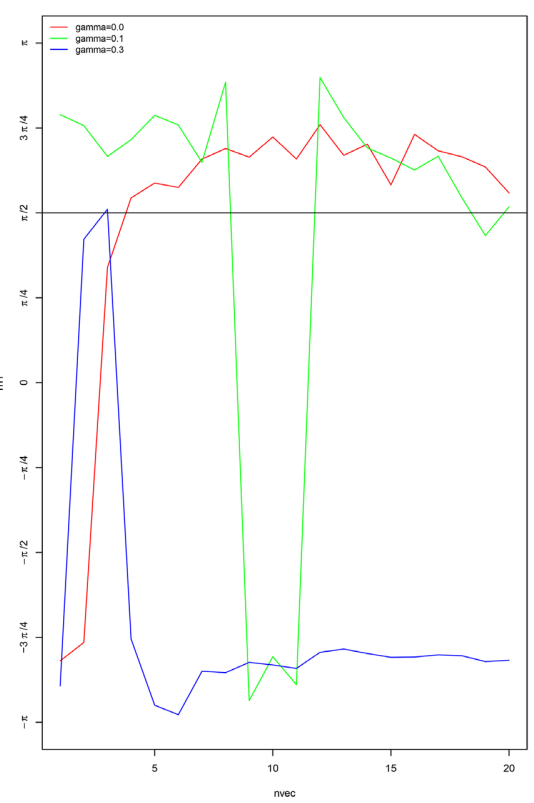

Figure 2. Rotangle against contours for fixed compression and varying gamma for one simulation.

\subsubsection{Interpretation for Figure 3}

From our plot, we observed that as the strength of compression increases from $c$ $=1.0$ up to $c=0.5$ for each contour level $l$, the average of the rotangle is around the marked rotangle at $\frac{\pi}{2}$. Also down the plot as the level of contour increases from $l=5$ up to $l=30$, our box plots become shorter and hence the estimated contours look more elliptical. This means that at high level of contours and at high strength of compression, the contours are informative. The lower level contours look spherical in all parameters. 

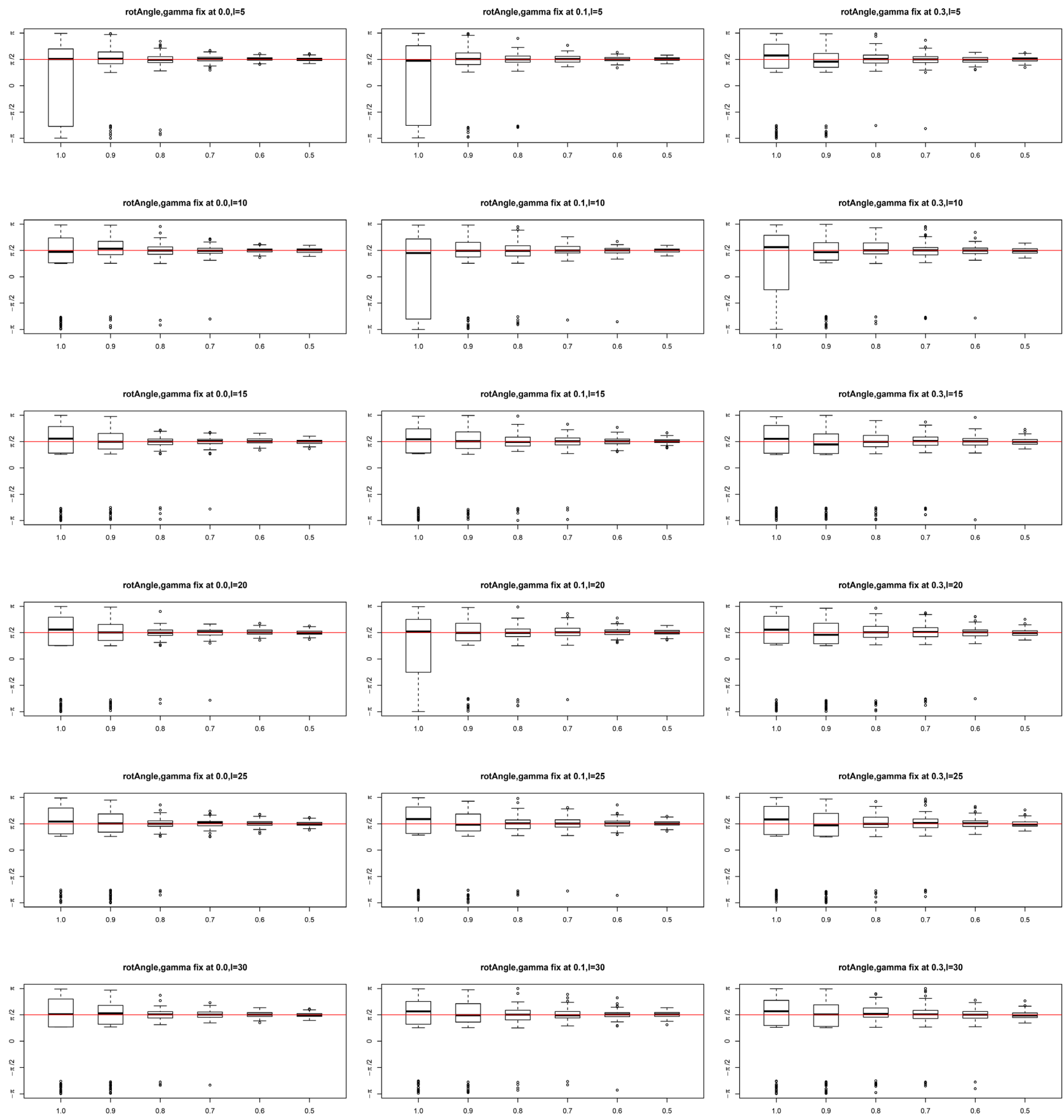

Figure 3. Rotangle against contours for fixed gamma and varying compression for 100 simulations.

\subsubsection{Rotangle, Fixing Compression and Varying Gamma for 100 Simulations}

Now we proceed by fixing the strength of compression $c$ and vary the regularity parameter $\gamma$. We simulated 100 times and again we chose the contour level $l=$ $5,10,15,20,25,30$. The box plot in Figure 4 below summarises our results.

\subsubsection{Interpretation for Figure 4}

From our plot, we observed that for each contour level $l$, as the regularity para- 
meter $(\gamma)$ of the point process decreases from 0.0 to 0.3 , our box plots become taller and thus the overall rotangle deviates from the marked rotangle at $\frac{\pi}{2}$. Again, at low strength of compression say at $c=0.9$ and irrespective of the contour level $l$, the box plot becomes taller meaning the overall rotangle deviates from the marked rotangle. This means that in most regular point process and at high strength of compression, the contours are more informative.
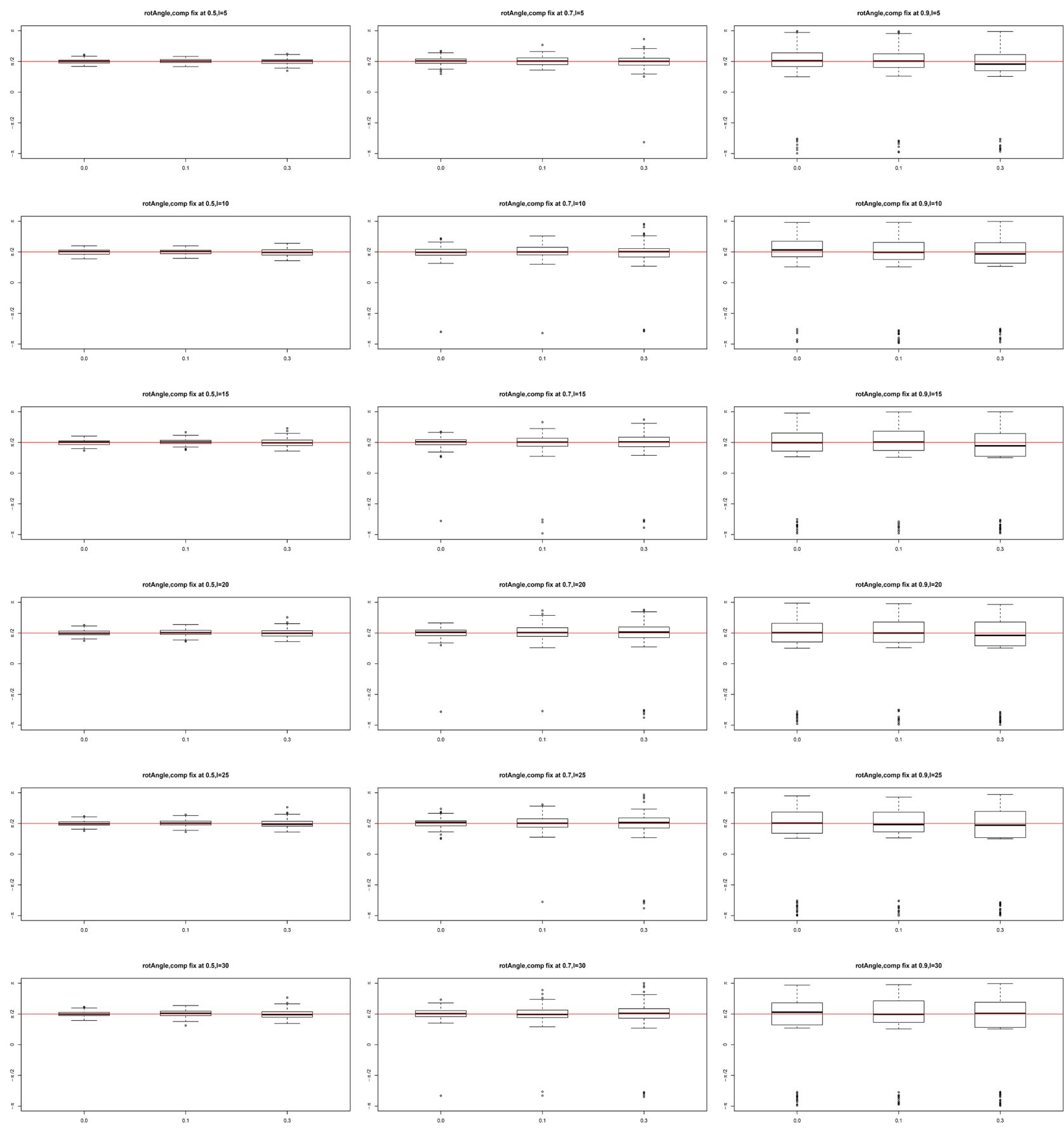

Figure 4. Rotangle against contours for fixed compression and varying gamma for 100 simulations. 


\subsection{P-Value Test of Equality of the Semi Axes}

The p-value test of equality of the semi-axes is simply a statistical test that tests if the semi axes of the ellipse in the Fry plot are equal. The contrast of interest (null hypothesis) is that the semi axes are equal $\left(a_{1}-a_{2}=0\right)$ where $a_{i}$ is the length of ith semi-axes (in 2D). Let's assume that the null hypothesis is true and the value of the test statistic has being calculated. If $\mathrm{p}$-value is higher than $\alpha$ then it means we can accept equality which means that the ellipse is in reality a ball meaning there was no compression. If there is compression and equality is accepted, then the chosen contour is not informative. If the p-value is less than (or equal to) $\alpha$, we reject the null hypothesis in favour of the alternative hypothesis. $\left(a_{1}-a_{2} \neq 0\right)$. We tested at $5 \%$ level of significance in all simulations $(\alpha=0.05)$.

\subsubsection{P-Value, Fixing Gamma and Varying Compression for One Simulation} We fixed the regularity parameter of the point process and the strength of compression was being varied for one simulation. We plotted the p-value against the levels of contour. The significance level $\alpha=0.05$ was indicated with a black horizontal line and different colours were chosen for different strength of compression. Figure 5 below summarises our results.
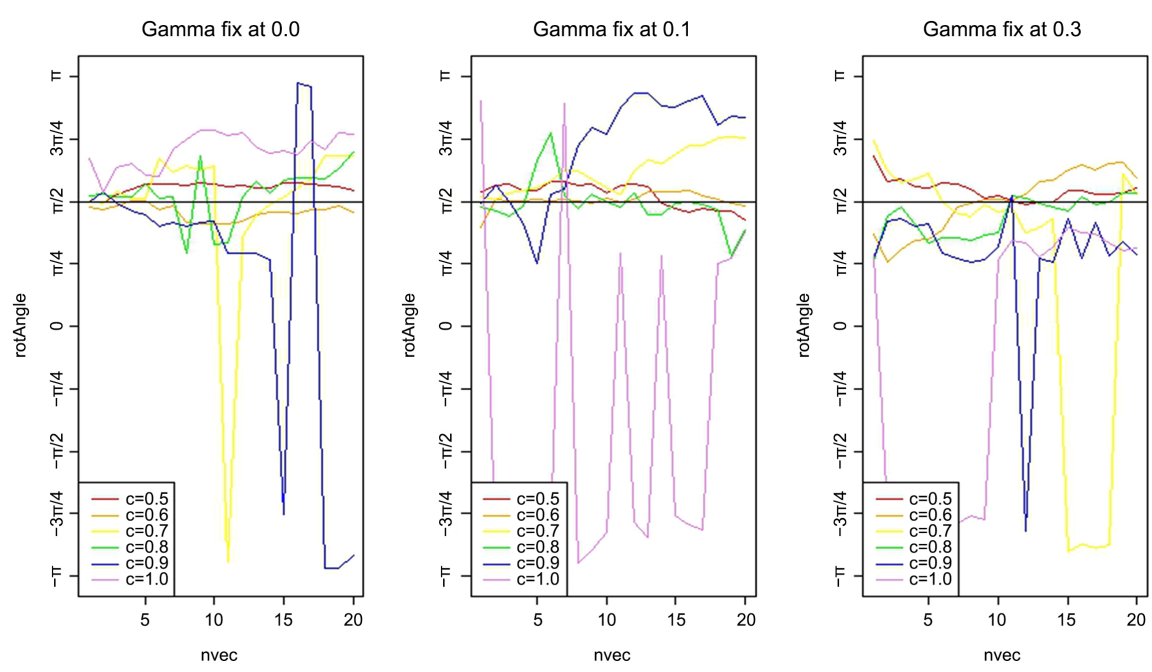

Figure 5. P-value against contours for fixed gamma and varying compression for one simulation.

\subsubsection{Interpretation for Figure 5}

From our plot and for each fixed value of gamma, we observed that at high strength of compression (say $c=0.5$ and $c=0.6$ ) and as the level of contour increases, the smaller the p-value (below $\alpha=0.05$ ). This means that at high strength of compression and at high level of contour, we reject the null hypothesis that the semi axes of the estimated ellipsoids are equal and hence the contours are informative in these parameters. Also, the p-value increases as we vary the regularity parameter from 0.0 to 0.3 meaning the contours in the least regular patterns are not informative. Low level contours are not as informative as the high contour levels in all parameters. 


\subsubsection{P-Value, Fixing Compression and Varying Gamma for One Simulation}

Now we fixed compression and vary the regularity parameter of the point process and with the same level of significance at $\alpha=0.05$ and the same number of contours (nvec $=1: 20$ ). The plot in Figure 6 below summarises our results.
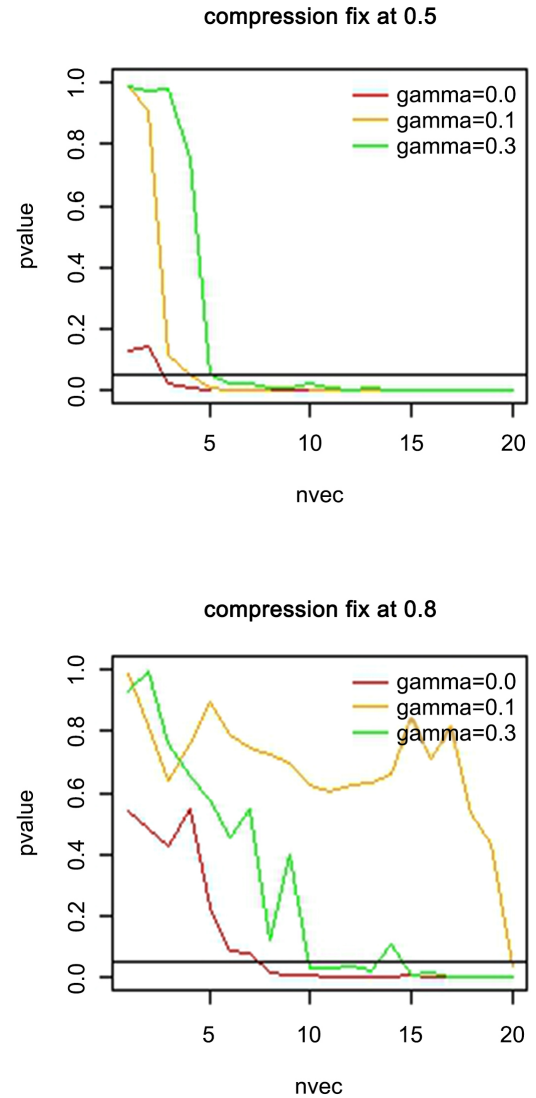
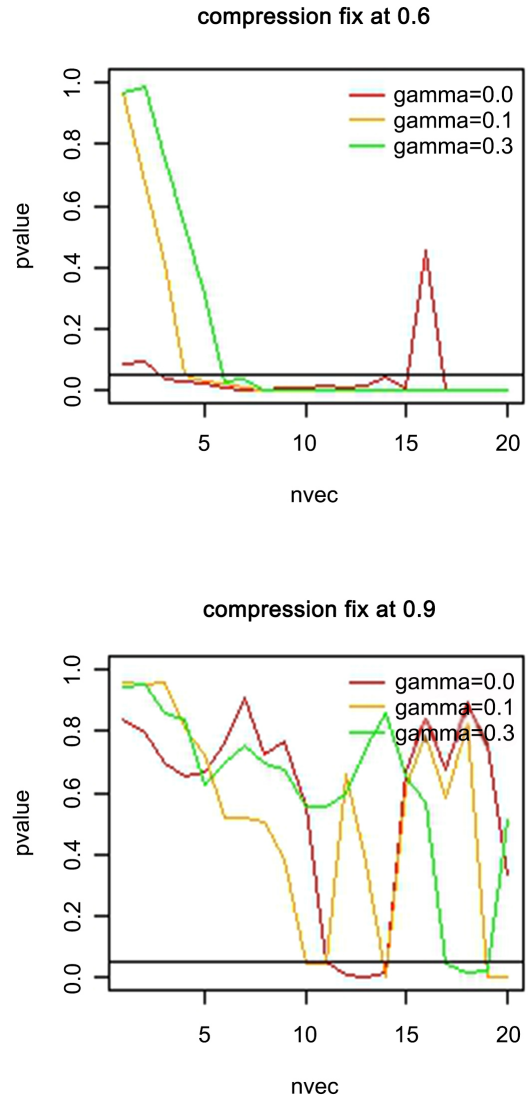
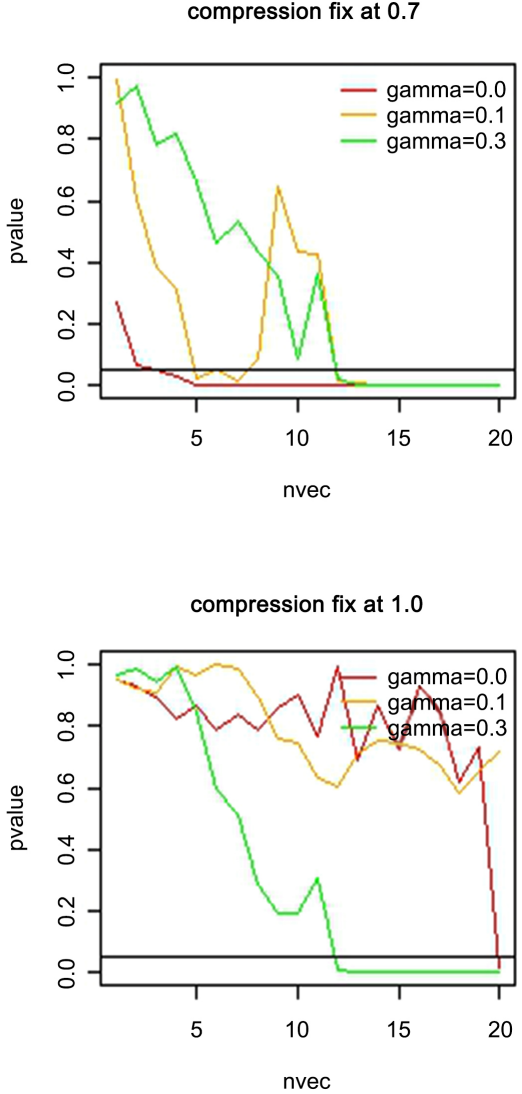

Figure 6. P-value against contours for fixed compression and varying gamma for one simulation.

From our plot and for each fix value of compression, we observed that at high level of contour and in more regular case $(\gamma=0.0$ and $\gamma=0.1)$, the p-value of the test decreases (below $\alpha=0.05$ ). This means that we can reject the null hypothesis that the semi axes of the estimated ellipsoids are equal in these parameters and hence the contours are informative. Again, at low strength of compression $(c=$ $0.8,0.9,1.0)$, the $p$-values increase and therefore accepting equality of the semi axes of the estimated ellipsoids in these parameters. For $c=1$ (no compression), the test confirms the correct shape of balls/spheres as expected (isotropic case).

\subsubsection{P-Value, Fixing Gamma and Varying Compression for 100 Simulations}

Now we fixed gamma and compression was being varied. We chose the contour levels $l=5,10,15,20,25,30$ due to high number of contours and the simulation was done 100 times. We tested at 5\% $(\alpha=0.05)$. The box plot in Figure 7 below summarises our results. 

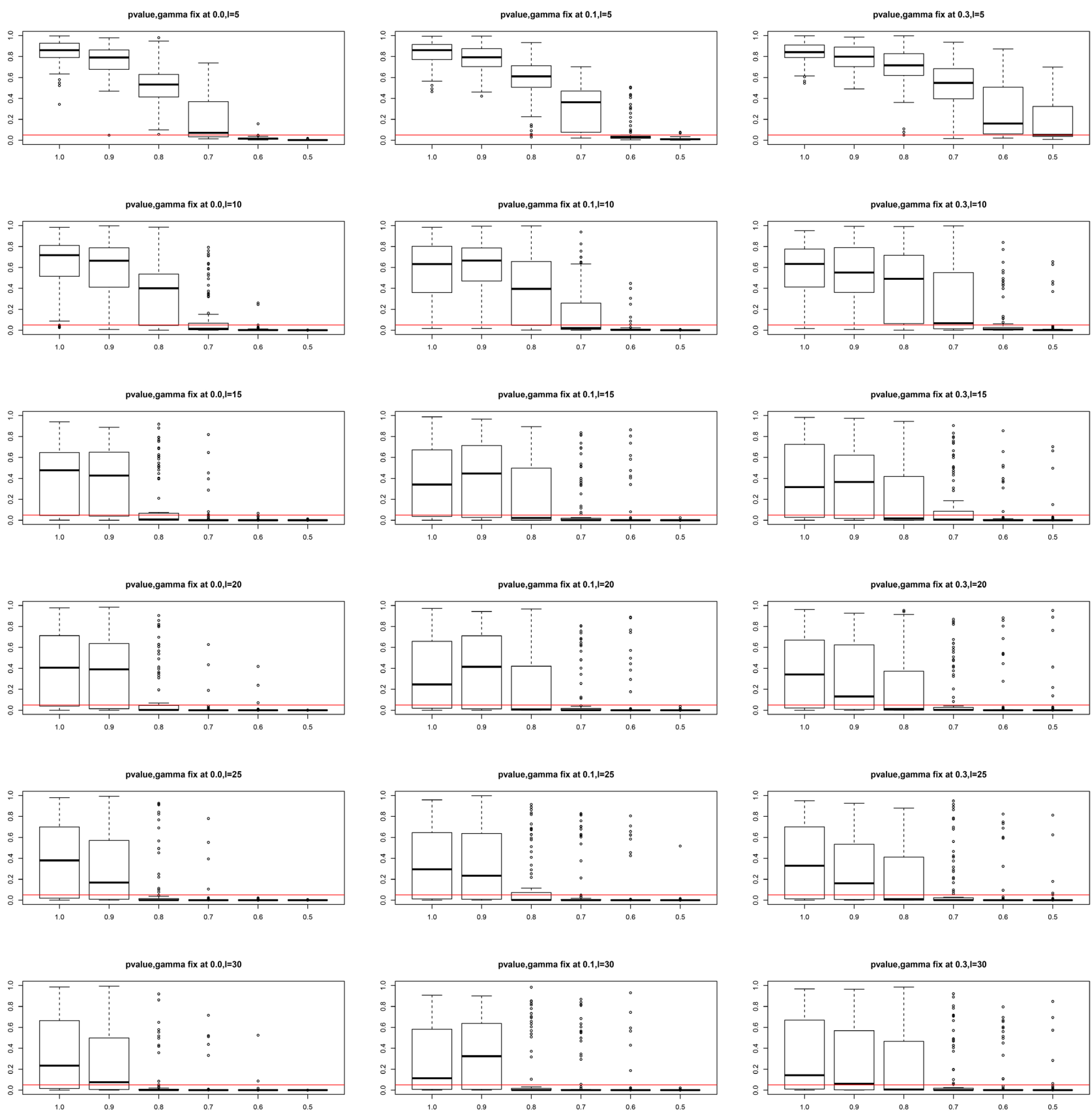

Figure 7. P-value against contours for fixed gamma and varying compression for 100 simulations.

\subsubsection{Interpretation for Figure 7}

From our plot, we observed that for each fix level of contour, as the strength of compression increases from $c=1.0$ to $c=0.5$, the overall $\mathrm{p}$-values of the test decrease. Further, as we go down the plot and as the level of contour increases from $l=5$ to $l=30$, the overall $\mathrm{p}$-value also decreases. This means that at high strength of compression and at high level of contours, we reject the null hypothesis that the semi axes of the estimated ellipsoids are equal and hence the contours are informative in these parameters. Further, as we move across the plot for each fixed level of contour and as we decrease the regularity of the point 
process, the overall $\mathrm{p}$-value increases and hence the contours are not informative in these parameters.

\subsubsection{P-Value, Fixing Compression and Varying Gamma for 100 Simulations}

Now we fixed compression and gamma was being varied. We chose the contour levels $l=5,10,15,20,25,30$ due to high number of contours and the simulation was done 100 times. We tested at 5\% ( $\alpha=0.05)$. The box plot in Figure 8 below summarises our results.
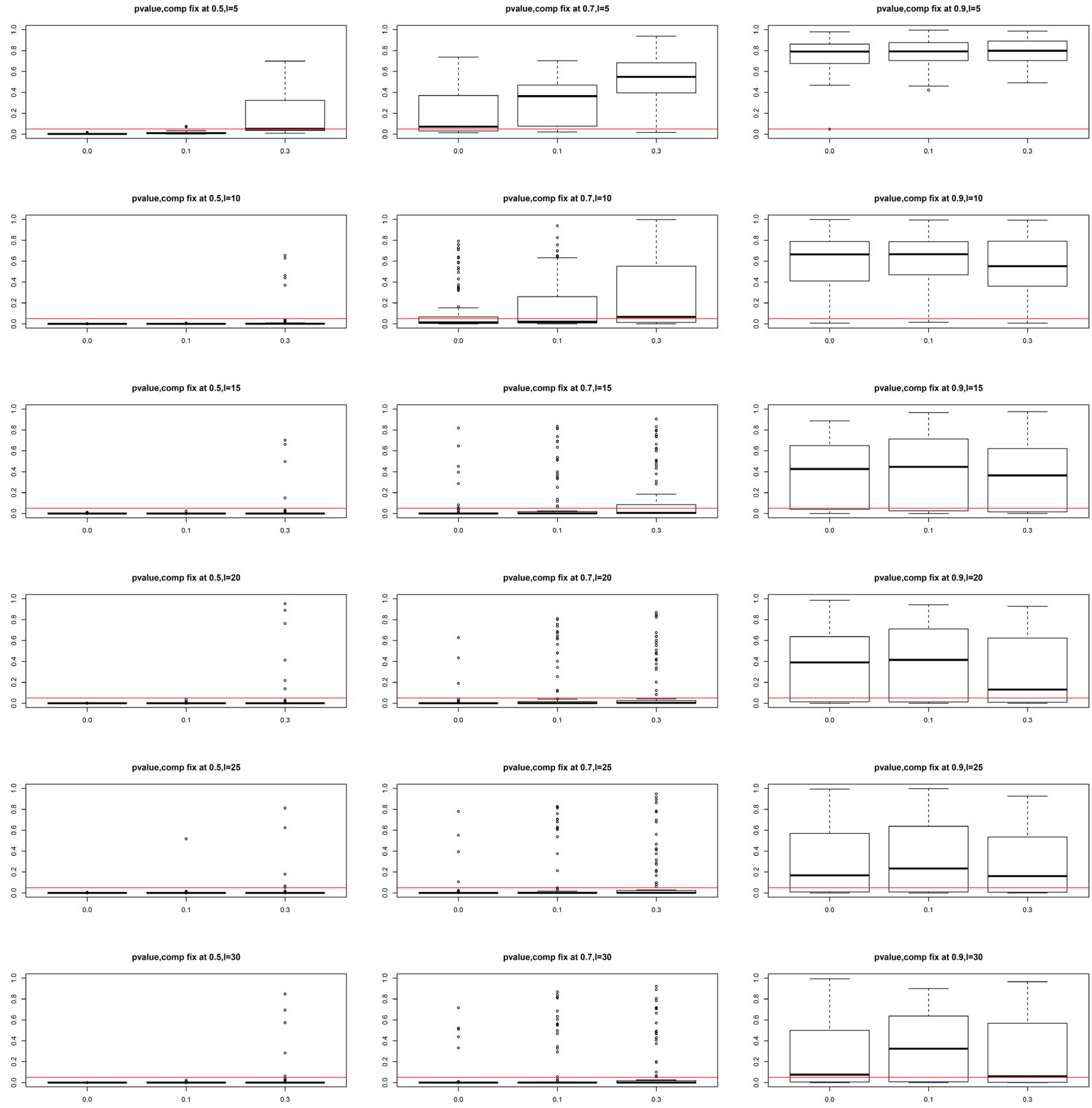

Figure 8. P-value against contours for fixed compression and varying gamma for 100 simulations. 


\subsubsection{Interpretation for Figure 8}

From our plot, we observed that for each fix level of contour, the p-value of our test increases as the regularity of the point process decreases. Also as we go down the plot and as the level of contour increases for fix strength of compression, the overall p-value also decreases. This means that in more regular point pattern and at high level of contour, we reject equality of the semi axes of the estimated ellipsoids and hence the contours are informative in these parameters. As we move across the plot and as the strength of compression decreases for fix level of contour, the overall p-value increases and hence the contours are not informative in these parameters.

\section{Findings}

In theory, the estimated ellipsoids should be more spherical as the level of contour increases [1] [2] The higher contours look more spherical because the anisotropy information gets weaker (it smoothes out) as we get further away from the origin in the Fry points but that was not the case in practice. In all the measures (rotangle and p-value test of equality), we considered for our simulation, we found out that low level contours are not informative as expected but high levels of contour are informative. This is to say the higher levels of contours should be considered, lower levels of contour are not informative and that one can ignore the very few lower contours and start from say the 5 th level of contour.

Again, in all our results, we found out that at low strength of compression ( $c=$ $0.8,0.9,1.0)$, the estimated ellipsoids get more spherical as expected. At high strength of compression ( $c=0.5,0.6,0.7)$, we found out that our estimated ellipsoids are informative rendering our contours meaningful in these set of parameters. $c=0.5$ is the best case.

Furthermore, in all our results, we found out that as the regularity of the point process increases $(\gamma=0.0,0.1)$, the estimated ellipsoids get more elliptical and hence the chosen level of contour is informative in these set of parameters. $\gamma=$ 0.0 is the best case. In the least regular case $(\gamma=0.3)$, the estimated ellipsoids look more spherical and hence the contours are not informative in this parameter. In summary, we found out that high levels of contour should be considered in all parameters.

\section{Conclusion}

In conclusion and due to the model we considered, at high level of contour coupled with high strength of compression $(c=0.5,0.6,0.7)$ and in more regular point process $(\gamma=0.0,0.1)$, our estimated ellipsoids are more elliptical (not like sphere) and hence these parameters are good for estimating anisotropy geometrically by the method of Fry. At low level of contour coupled with low strength of compression $(c=0.8,0.9,1.0)$ and in low regular point process $(\gamma=0.3)$, the estimated ellipsoids look more spherical like expected. 


\section{Conflicts of Interest}

The author declares no conflicts of interest.

\section{References}

[1] Thomas, A.R., Aila, S., Claudia, R. and Martina, S. (2016) Estimating Geometric Anisotropy in Spatial Point Patterns. Spatial Statistics, 15, 100-114. https://doi.org/10.1016/j.spasta.2015.12.005

[2] Møller, J. and Toftaker, H. (2014) Geometric Anisotropic Spatial Point Pattern Analysis and Cox Processes. Scandinavian Journal of Statistics, 41, 414-435. https://doi.org/10.1111/sjos.12041

[3] Claudia, R., Aila, S., Johannes, F. and Katja, S. (2009) Anisotropy Analysis of Pressed Point Processes. AStA Advances in Statistical Analysis, 93, 237-261. https://doi.org/10.1007/s10182-009-0106-5

[4] Guan, Y., Sherman, M. and Calvin, J.A. (2006) Assessing Isotropy for Spatial Point Processes. Biometrics, 62, 119-125. https://doi.org/10.1111/j.1541-0420.2005.00436.x 